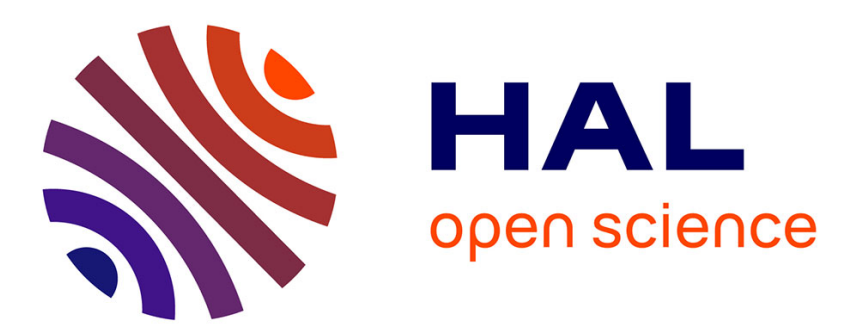

\title{
Polygenic Adaptation: Integrating Population Genetics and Gene Regulatory Networks
}

Maud Fagny, Frederic Austerlitz

\section{To cite this version:}

Maud Fagny, Frederic Austerlitz. Polygenic Adaptation: Integrating Population Genetics and Gene Regulatory Networks. Trends in Genetics, 2021, 37 (7), pp.631-638. 10.1016/j.tig.2021.03.005 . hal03100982

\section{HAL Id: hal-03100982 \\ https://hal.science/hal-03100982}

Submitted on 6 Jan 2021

HAL is a multi-disciplinary open access archive for the deposit and dissemination of scientific research documents, whether they are published or not. The documents may come from teaching and research institutions in France or abroad, or from public or private research centers.
L'archive ouverte pluridisciplinaire HAL, est destinée au dépôt et à la diffusion de documents scientifiques de niveau recherche, publiés ou non, émanant des établissements d'enseignement et de recherche français ou étrangers, des laboratoires publics ou privés.

\section{(ㅇ)(1) $\$$}

Distributed under a Creative Commons Attribution - NonCommercial - NoDerivatives| 4.0 


\title{
Understanding the adaptation of polygenic traits: the importance of gene regulatory networks
}

\author{
Maud Fagny ${ }^{\mathrm{a}}$, Frédéric Austerlitz ${ }^{\mathrm{a}}$ \\ ${ }^{a}$ UMR7206 Eco-Anthropologie, Muséum National d'Histoire Naturelle, Centre National de la Recherche \\ Scientifique, Université de Paris, Paris, France
}

\begin{abstract}
The adaptation of populations to local environments often relies on the selection of beneficial versions of polygenic traits. At the molecular level, this process corresponds to polygenic selection models, in which many independent loci, often located in regulatory regions, experience subtle shifts in frequency. Here, we first review the different approaches developed to detect signatures of polygenic selection, their power and limitations. We then highlight the contribution of systems biology to the understanding of the molecular bases of polygenic traits and the evolution of gene regulatory networks involved in these traits. Finally, we discuss the need for a unifying framework merging the fields of population genetics, quantitative genetics and systems biology to better understand the molecular bases of polygenic traits adaptation.

Keywords: Quantitative traits, Polygenic selection, Gene regulatory networks, Systems Biology, Adaptive evolution, Population genetics modelling
\end{abstract}

\section{Highlights}

- In many species, adaptation of populations to local environment relies on traits that have a polygenic architecture.

- Statistical approaches have been developed recently to specifically detect different aspects of the weak genomic signatures left by directional polygenic selection.

- Genome-wide scans for selection have revealed that polygenic selection is a driving force of local adaptation and disproportionately targets regulatory regions, hinting for an important role of gene regulatory networks in evolution.

- Gene regulatory network inference helps identifying and grouping together genes and regulatory elements that participate to the same biological processes. 
- The topology of gene regulatory elements put constraints on which genes and regulatory regions can be leveraged by polygenic selection.

\section{Local adaptation of polygenic traits: the importance of regulatory regions}

Many adaptive phenotypes - i.e. phenotypes involved in adaptation to local environments - are polygenic (see Box 1). Also called quantitative or complex traits, these phenotypes are determined by multiple genes and regulatory loci. This complex genetic architecture has strong implications on how these traits evolve. If these traits are under selection (either stabilising or directional), the underlying genetic model will be a model of polygenic selection, i.e. subtle shifts in frequencies at many independent loci coding for each adaptive trait [1-3]. This is for example the case for height, immune response, and metabolism in humans $[4,5]$, flowering time, and stress response in plants, [6-8], or pathogenicity in yeast [9]. Moreover, in humans, a lot of disorders such as autoimmune diseases and metabolic disorders are considered to be consequences of past adaptation of polygenic traits to changes in local environments, such as pathogens or lifestyle transition [10, 11]. Being able to detect polygenic adaptation is thus important not only to understand the evolution of polygenic traits, but also the origin of many human diseases.

Our apprehending of the effects of directional selection on polygenic traits at the molecular level has strongly improved in the past ten years. QTL and genome-wide association studies (GWAS), have led to the identification of genetic variants associated with these polygenic traits, which were located overwhelmingly in non-coding regions $[12,13]$ - up to $90 \%$ in some species - highlighting the importance of regulatory regions. Population genetics studies, leveraging the ever growing number of genomic data sets available and the improvements in genomic functional annotation have confirmed that local adaptation often relies on selection of mutations located in regulatory regions that impact gene expression levels $[9,14]$. This has spurred efforts to develop population genetic approaches to detect signatures of selection beyond coding genomic regions. Independently, these results helped creating new theoretical models, in which polygenic traits result from a complex interplay of regulatory relationships between genes and different molecular actors at the cellular level. This led to the development of systems biology models aiming at understanding how these regulatory relationship evolve under directional selection, which brought new clues to understand the molecular bases of polygenic traits adaptation to local environments. 


\section{Detecting genomic signatures of polygenic selection: an ongoing quest}

During the past few years, population geneticists have attempted to develop approaches to detect signatures of polygenic selection on genomic data. The first difficulty is to identify the type of signatures that one must look for. For this purpose, models have been developed to investigate the expected impact of polygenic adaptation on population genomic data [3, 15-19] (see Box 2). They assume additive and/or epistatic interactions among loci. These studies have shown mainly that polygenic adaptation is likely to yield subtle frequency shifts at the genes involved in the polygenic trait: polygenic adaptation events target several mutations simultaneously and leave weak molecular signatures on the genome [20]. Recently, Hollinger and colleagues have however shown that the type of genomic signature observed ranges from sweeps (either total or partial) to subtle frequency shifts, depending on the population-scaled mutation rate [3].

Methods to detect genomic signatures of polygenic selection events have mainly focused on combining signatures of positive selection across sets of mutations that are defined a priori as likely candidates for adaptive pressures. In particular, some studies have proposed to group mutations for which allele frequencies correlate with specific ecological variables [4, 21]. These approaches, while powerful, require access to past and present environmental data for the populations under study, which are often difficult to obtain. Other approaches use gene pathways and information about biological functions or GWAS results to group mutations together [5, 8, 22]. The first approach consists in investigating whether known pathways or gene sets are particularly enriched in selection signals [5], with many loci exhibiting a high level of genetic differentiation among populations. A recently developed related approach looks for local subnetworks under strong differentiation [23].

The second approach consists in testing whether a known trait has been submitted to divergent selection [22, 24-26]. These methods require that previously performed genome-wide association studies (GWAS) are available, which have detected loci associated with the traits and estimated their additive effect on these traits. This allows computing the polygenic score of the genotyped individuals in the studied populations, which can then be compared to what is expected under genetic drift only. Because they rely on already existing GWAS studies, these methods cannot be used blindly on the genome.

On the other hand, other methods have focused on scanning the genome in order to detect loci with high level of differences in allele frequencies among populations, without a priori grouping mutations. This is the case for example of the SDS approach [27], OutFLANK [28] and PCAdapt 
[29]. These genome-scan methods can be used blindly on the genome, however they were mainly developed in a context of strong selection on a single locus and simulation studies have shown that their efficiency for detecting loci evolving under polygenic selection is rather limited [30, 31].

\section{Shortcomings of polygenic selection detection approaches}

The shortcomings of existing approaches to detect polygenic selection have been extensively discussed in recent papers [32-35] that have raised several caveats concerning the results of these polygenic selection tests. The first set of caveats are technical and relate primarily to GWAS-based results: if not correctly taken into account, a hidden population structure in the samples used for GWAS can lead to the false detection of directional polygenic selection signal on the trait under study. This has been demonstrated for height in European human populations [34, 35]. Similarly, GWAS ascertainment biases can confuse selection signals [36]. Transferring GWAS results across time and space can also lead to misidentification of causal mutations, the impact of which on the selection tests is uncertain.

The last set of caveats relates to the interpretation of test results. They include the potential existence of gene $\mathrm{x}$ environment interactions and the poorly known interactions between traits [32], both of which can hide the selection signals or inverse the direction of selection. Finally, an important part of the selection signals might be lost in current studies because a fundamental component of the genetic architecture of polygenic traits is often minimized or totally ignored: epistatic interactions [37]. On this topic, Payne and colleagues recently highlighted the importance of studying the topography of the adaptive landscape and, in particular, the exceptional characteristics of gene expression regulatory networks in terms of epistasic interactions, pleiotropy and robustness [38].

To this list of caveats, we can add another one: while carrying the majority of the genetic variation associated with polygenic traits, the regulatory regions are a blind spot of the methods designed to detect polygenic selection described above. These approaches, which combine signatures of positive selection across loci associated to a given trait, often only target genes, completely ignoring regulatory regions. The recently developed approaches based on GWAS results include some genetic variants from regulatory regions. However, because they only include the variants that are statistically significantly associated to the phenotype at the genome-wide level, they miss a good part of the genetic bases of polygenic phenotypes - the famous "missing heritability" [39, 40]. 
On the other hand, blind genome-wide scans have detected the signatures of polygenic selection in regulatory regions. Several studies have indeed revealed an enrichment in positive selection signals among cis-regulatory elements and expression QTLs [41-43]. However, the lack of functional annotation of these regions often prevents identification of targeted phenotypes, beyond general results of Gene Ontology enrichment assays.

In short, despite recent advances in polygenic selection signature testing, several difficulties persist. While the technical caveats point to the need to carefully consider potential confounding effects and to assess the sensitivity and specificity of polygenic selection tests, most of the difficulties in interpreting polygenic selection tests can be explained by the "genomic only" approaches used in these tests. These difficulties indeed arise from our limited knowledge of the true genetic architecture and molecular bases of polygenic traits. These traits are determined by various regulatory regions, and depend on a complex interplay between different actors that are expressed following a rigid spatio-temporal framework. Ignoring these features makes it difficult to interpret results of polygenic selection tests, and to identify the biological functions and traits potentially under polygenic selection.

\section{Embracing the Complexity: Contributions from Systems Biology}

Organizing and integrating information from diverse sources and understanding how polygenic phenotypes are generated from the genome at different levels, from the cell to the organism, is the core of systems biology [44]. The rise of this research field initiated a switch in the perception of the molecular bases of polygenic traits, from a gene-first to an interaction-first model. Quantitative genomics apprehends indeed the molecular bases of polygenic phenotypes as a collection of independent genes, each coding for a fraction of the phenotype, with potentially some interactions. Conversely, systems biology focuses primarily on these interactions, modelling the regulatory relationship between genes, their regulators and their products. During the past twenty years, buoyed by the availability of high-throughput data, numerous approaches have been developed to model these regulatory relationships and to efficiently deal with their complexity. In particular, the representation of these relationships as Gene Regulatory Networks (GRNs), which gather tens to thousands of genes, has allowed better understanding the molecular bases of polygenic traits [45]. From the systems biology point of view, understanding the molecular bases of polygenic selection consists, therefore, in deciphering how regulatory interactions among genes evolve under 
constraints. Models have been developed to study how GRNs evolve, which explicitly simulates these interactions. Initially developed by Wagner, they model gene networks through a matrix whose coefficients determines how each gene regulates each other and itself [46, 47]. These coefficients vary among individuals and can evolve through mutations that change the regulatory relationships between genes. Each matrix leads to a given level of expression of each locus. Selection will occur through the assumption that there is an optimal level of expression for each gene. This model was first developed to study the evolution of gene duplications [46].

It allowed then discovering some properties of GRN evolution. One of the major results is the demonstration that, under stabilizing selection, network evolves toward a state where a single mutation becomes less likely to reduce the fitness of the individuals [47], a phenomenon called canalisation [48]. This insensitivity to mutations becomes stronger when the number of connections in the network increases [49] or when phenotypic optima become more extreme [50]. Conversely, this robustness of the network promotes the accumulation of hidden loss-of-function mutations, which can then accelerate adaptation in a changing environment [51].

GRN evolution models have also helped better understanding how the structure of these GRNs impacts the individual response of each gene to selection, as a function of selection intensity and distance between the current phenotype and the optimal one. Genes that are more upstream in the network (i.e. strongly regulating the other genes and/or less regulated by them) are more affected by selection [52]. This could explain the empirical observation that transcription factors are evolving faster than the other genes [53].

\section{Impact of GRN structure on architecture of polygenic selection}

Understanding the evolution of GRNs that underlies polygenic phenotypes is currently a hot topic in evolutionary biology. The various scenarii of GRN evolution observed have been summarized for animals, insects [54] and plants [55]. Modifications of GRNs seems to be frequent during speciation [56] and domestication [57, 58], and can lead to dramatic changes in developmental patterns and other polygenic phenotypes. Moreover, there are many examples of directional and polygenic selection events affecting the gene regulatory networks [8, 9, 43, 59]. The analysis of cellular GRN and their evolution has led to hypotheses about how polygenic selection can act, and how gene regulatory network structure might facilitate or constrain it. Three GRN features are worthy of attention in this context: redundancy, modularity, and their scale-free property (see 
Figure 1 and Box 3).

Redundancy is a characteristics of GRNs that ensures stability of gene expression, buffers noise and ensures phenotype robustness to random mutations that may cause gene inactivation [50, 60]. It has been shown also to facilitate polygenic adaptation. For example, an evolution experiment in Drosophila showed phenotypic convergence in response to a temperature change in ten populations, while the genetic analysis revealed that different pathways were targeted by polygenic selection in each replicate [61]. Redundancy of GRNs thus facilitated polygenic selection by increasing the number of available targets to reach the optimum phenotype (see Figure 1B). However, this polygenic selection will be difficult to detect, as it will potentially affect different pathways among populations confronted to the same constraints.

GRNs are also highly modular, i.e., made of small groups of genes strongly interconnected with each other but only loosely connected to the rest of the network, thus constituting a module. It has been proposed, in the omnigenic model, that as all genes are seemingly interconnected within the GRN, each trait might be influenced by all genes [40]. Theoretically, such extreme generalized pleiotropy - property of a gene that influences several, apparently unrelated traits - would severely constrain the possibility for polygenic selection to act, because it could not modify a given trait without influencing all the others. However, the modularity of GRNs can mitigate this effect. Indeed, in each module, only a few genes are connected with genes from other modules. Most genes in a module are thus isolated from the rest of the network and can be leveraged by polygenic selection (see Figure 1A).

Finally, GRNs tend to be scale-free, i.e. there are many more genes that are connected to only a few other genes than there are genes connected to lots of other genes [62] (see Figure 1B). This has been shown on various types of networks $[45,62,63]$. As confirmed by observations on molecular data (transcriptomics or proteomics), the level of connection of a gene in a network is inversely correlated with its level of genetic diversity [64-66]. This means that the handful of highly pleiotropic genes at the center of these networks often evolve under strong negative selection and present few variations of expression among individuals. Conversely, the vast majority of genes are at the periphery of the network, involved in only one or few pathways. Changes in their regulation will, therefore, only very slightly affect the other pathways (see Figure 1A and B). This leaves open a large field full of candidates for polygenic selection to act on, both at the level of the gene sequence and the gene regulatory elements, while limiting the potential side effects of selection on 
other pathways.

\section{Polygenic selection and GRNs: advantages and limits}

Theoretical models of GRNs combined with results on real data sets allow better understanding the molecular bases of polygenic phenotypes and their evolution. They also help understanding where and how polygenic selection can act, and where to look for genomic signatures of selection. However, the models often focus on the evolution of unipartite networks of genes interacting with each other, thus overlooking important features that would be useful to get a complete picture of how polygenic selection can impact GRNs. First, despite the evidences for pervasive polygenic selection targeting regulatory mutations [9], theses models do not include explicitly how modifications in non-coding regulatory regions, such as enhancers, can impact the expression level of several genes at once, while not modifying their interactions. An important source of polygenic trait variation and evolution is thus not taken into account. Some current approaches integrate heterogeneous data using multipartite networks, in order to identify not only gene-gene regulatory interactions but also interactions between regulatory regions and genes [45, 67, 68]. These approaches could serve as bases for the development of new GRN evolution models. Second, most studies on GRN evolution do not model individual nucleotides. Consequently, they allow to study questions such as redundancy, pleiotropy and canalisation, but not to dissect the genetic bases of evolution, and to understand the genomic signatures that may be expected in different polygenic selection scenarios.

\section{Concluding Remarks and Future Perspectives: towards systems population genetics}

In order to detect polygenic selection and to disentangle the impact of several confounding factors, we need to better understand not only the genetic architecture of polygenic traits, but also their molecular architecture. A new integrative evolutionary framework becomes quite indispensable, which would take this architecture into account, in particular its redundancy and the fact that two selective events in the same population might not lead to the same selection scenario at the molecular level [69]. Here, we argue that, in order to achieve such a unifying framework, we need not only to identify which genes and pathways contribute to polygenic traits, and how redundant they are, but also investigate the complex regulatory interactions that govern their expression (see Outstanding Questions). In particular, we need to take into account how the genomic regulatory 
regions impact the expression of these traits. We should focus on the crucial post-trancriptional regulatory steps, on the gene-gene interactions, and maybe more importantly, on the position of these genes in the cellular gene regulatory network. All of this could be achieved by combining population genomics and system biology approaches.

\section{Outstanding Questions}

- Redundancy allows for the selection of the same version of a trait through the selection of genetic variants in different pathways. Do we observe this phenomenon at the scale of the population? In that case, which molecular signatures do such evolutionary processes leaves on the genome and how can we detect them?

- Pleiotropy is a major property of gene regulatory networks actors that constrains how polygenic selection acts. Genes are often involved in several distinct pathways. What is the level of pleiotropy of the regulatory elements, in particular of the distal ones such as enhancers and how does it constrain their evolution?

- Classical population genetics approaches model the evolution of genetic sequences but ignore their complex interactions in gene regulatory networks. On the other hand, gene regulatory networks model the evolution of these regulatory relationships but not their genetic bases. Will more integrated approaches that explicitly model the molecular evolution of regulatory regions in the context of gene regulatory networks improve our understanding of the molecular bases of local adaptation?

- Selection on polygenic traits yields linkage disequilibrium among genes involved in the traits, at the within- and among-population levels [15]. How do regulatory interactions affect this linkage disequilibrium? Can it be used to detect polygenic selection?

- GWAS-based method aiming at detecting polygenic selection use the additive effect of each gene on the trait. Could the interaction (dominance, epistasy) between these genes also be integrated in these approaches? 


\section{Box 1. Genetic architecture of traits: from the Mendelian to the omnigenic model}

Polygenic traits have been the focus of many theoretical and empirical studies since Fisher's seminal model of polygenic inheritance, which bridged the gap between the Mendelian inheritance model and the observation of continuous traits. This model suggests that the apparent continuity of many phenotypic traits can be explained if multiple genes contribute additively to these traits [70]. Classic genetic studies, recent advances in quantitative genetics and genome-wide association studies (GWAS) have demonstrated the existence of a continuum of genetic architectures for traits, in many organisms including yeast, insects, worms, plants and mammals [39, 71-73]. These architectures range from the Mendelian model, where a trait is determined by a single gene (e.g. ABO blood groups in primates, Mendel's peas color and shape) to the infinitesimal model [74] where a seemingly infinite number of loci are involved in the determinism of a trait (e.g. height, in both humans and plants $[24,75])$. This last concept has been highlighted in a recent paper [40] that labelled it the 'omnigenic' model. In between, we find a myriad of traits that present an oligogenic or polygenic determinism, such as flowering time in plants, tolerance to ethanol in yeasts or susceptibility to diabetes in humans [75-78].

\section{Box 2. Polygenic selection}

Polygenic selection consists in natural or artificial selection occurring on a trait coded by several loci. Under this framework, even when the phenotypic optimum differs strongly from the current average phenotype, populations can evolve toward this optimum without substantial changes in allelic frequencies [15]. Indeed, each locus can undergo only swift changes in these frequencies, while allelic associations will appear among the different loci, allowing to reach the phenotypic optimum. In this case, methods aiming at detecting signals of selective sweeps such as $i H S$ [79] or $n S L$ [80] will probably not detect any signal, as no locus is undergoing a strong shift in allelic frequencies. Note, however, that this will depend on the number of loci involved in the trait: if this number is low, each locus will respond more strongly in terms of allelic frequencies changes and some might be detected. Moreover, if among the loci coding for the trait, some are major loci with a high contribution to genetic variance, these loci are more likely to be affected by a strong shift in allelic frequencies $[16,17]$, and thus to be detected by selective sweeps tests.

Other approaches modeled the fate of a locus coding for a given trait, while all other loci coding for this trait were treated as background genetic variance [18]. They find that a selective 
sweep will be observed in some cases at the focal locus, depending upon its relative variance as compared to the background loci and also upon the shape of the selection function. In a model that considered also epistatic interactions, Hollinger and colleagues then showed that the probability of observing a sweep at a locus involved in a quantitative trait will depend mainly on a parameter called the population-scaled background mutation rate $\Theta_{b g}$, i.e. the product of the effective size of the population by the total mutation rate of all background loci [3]. Low values for $\Theta_{b g}$ will lead to the occurrence of selective sweeps at the focal locus, while high values will yield small polygenic allele frequency shifts. Several partial selective sweeps will be observed for intermediate values.

\section{Box 3. Gene Regulatory Networks}

Gene Regulatory Networks (GRNs) describe a collection of molecules that interact together to regulate the level of expression of genes, both as mRNA and proteins. These regulatory relationships can be direct or indirect, for example when a transcription factor increases another protein level through the activation of its coding gene transcription.

GRNs are often represented as graphs, mathematical objects that represent the elements (nodes) and the interactions between elements (edges). They can include one type of elements such as proteins (unipartite graphs), or several types of elements such as transcription factors, regulatory mutations and mRNAs (multipartite graphs). The study of the topology of these graphs allows better understanding how the elements interact together and how GRNs evolve.

GRNs, whether in yeasts, insects, plants or mammals, show some shared topological characteristics. Most importantly, they are scale-free. This means that very few of the nodes within the network are connected to many others - the key or master regulators - while most of the nodes are connected only to a few other nodes (see Figure Figure 1B). GRNs are also highly modular, i.e. organized into groups of nodes that are strongly interconnected but only loosely connected to the rest of the network, thus constituting a module. The modules often correspond to biological pathways.

The topology of the network also seems to constrain the evolutionary history of each node. In GRNs, the handful of high-degree genes, i.e. connected to many other genes, are often known to be pleiotropic, involved in many pathways, and to be very constrained [64], evolve under strong negative selection and present few variations of expression among individuals. Conversely, peripherical genes, which are only connected to few other genes, are often included in only one pathway. 
306 They are also more likely to evolve under positive selection. 


\section{References}

[1] Pritchard, J. K. and Di Rienzo, A. (2010) Adaptation - not by sweeps alone. Nat Rev Genet 11, 665-667.

[2] Fraser, H. B. (2013) Gene expression drives local adaptation in humans. Genome Research 23, 1089-1096.

[3] Höllinger, I. et al. (2019) Polygenic adaptation: From sweeps to subtle frequency shifts. PLOS Genetics 15, e1008035.

[4] Hancock, A. M. et al. (2011) Adaptations to climate-mediated selective pressures in humans. PLoS genetics 7, e1001375.

[5] Daub, J. T. et al. (2013) Evidence for polygenic adaptation to pathogens in the human genome. Mol Biol Evol 30, 1544-58.

[6] He, F. et al. (2016) The Footprint of Polygenic Adaptation on Stress-Responsive Cis Regulatory Divergence in the Arabidopsis Genus. Molecular Biology and Evolution 33, 20882101.

[7] Zan, Y. and Carlborg, O. (2019) A Polygenic Genetic Architecture of Flowering Time in the Worldwide Arabidopsis thaliana Population. Molecular Biology and Evolution 36, 141-154.

[8] Hämälä, T. et al. (2019) Gene Expression Modularity Reveals Footprints of Polygenic Adaptation in Theobroma cacao. Molecular Biology and Evolution 37, 110-123.

[9] Fraser, H. B. et al. (2011) Systematic Detection of Polygenic cis-Regulatory Evolution. PLOS Genetics 7, e1002023.

[10] Barreiro, L. B. and Quintana-Murci, L. (2010) From evolutionary genetics to human immunology: how selection shapes host defence genes. Nat Rev Genet 11, 17-30.

[11] Chouchani, E. T. and Kajimura, S. (2019) Metabolic adaptation and maladaptation in adipose tissue. Nature Metabolism 1, 189-200.

[12] Edwards, S. L. et al. (2013) Beyond GWASs: illuminating the dark road from association to function. Am J Hum Genet 93. 
[13] Rodgers-Melnick, E. et al. (2016) Open chromatin reveals the functional maize genome. Proceedings of the National Academy of Sciences 113, E3177-E3184.

[14] Enard, D. et al. (2014) Genome-wide signals of positive selection in human evolution. Genome Research 24, 885-895.

[15] Latta, R. G. (1998) Differentiation of allelic frequencies at quantitative trait loci affecting locally adaptive traits. Am Nat 151, 283-292.

[16] Le Corre, V. and Kremer, A. (2003) Genetic variability at neutral markers, quantitative trait loci and trait in a subdivided population under selection. Genetics 164, 1205-1219.

[17] Kremer, A. and Le Corre, V. (2012) Decoupling of differentiation between traits and their underlying genes in response to divergent selection. Heredity 108, 375-85.

[18] Chevin, L.-M. and Hospital, F. (2008) Selective sweep at a quantitative trait locus in the presence of background genetic variation. Genetics 180, 1645-1660.

[19] Stephan, W. (2016) Signatures of positive selection: from selective sweeps at individual loci to subtle allele frequency changes in polygenic adaptation. Molecular Ecology 25, 79-88.

[20] Pritchard, J. K. et al. (2010) The genetics of human adaptation: hard sweeps, soft sweeps, and polygenic adaptation. Current biology: CB 20, R208-215.

[21] Haasl, R. J. and Payseur, B. A. (2016) Fifteen years of genomewide scans for selection: trends, lessons and unaddressed genetic sources of complication. Molecular Ecology 25, 5-23.

[22] Berg, J. J. and Coop, G. (2014) A Population Genetic Signal of Polygenic Adaptation. PLoS Genetics 10, e1004412.

[23] Gouy, A. et al. (2017) Detecting gene subnetworks under selection in biological pathways. Nucleic Acids Res 45, e149.

[24] Turchin, M. C. et al. (2012) Evidence of widespread selection on standing variation in Europe at height-associated SNPs. Nat Genet 44, 1015-1019.

[25] Racimo, F. et al. (2018) Detecting polygenic adaptation in admixture graphs. Genetics 208, 1565-1584. 
[26] Robinson, M. R. et al. (2015) Population genetic differentiation of height and body mass index across Europe. Nature Genetics 47, 1357-1362.

[27] Field, Y. et al. (2016) Detection of human adaptation during the past 2000 years. Science 354,760 .

[28] Whitlock, M. C. and Lotterhos, K. E. (2015) Reliable detection of loci responsible for local adaptation: Inference of a null model through trimming the distribution of $F_{\text {st. }}$. Am Nat 186 Suppl 1, S24-S36.

[29] Duforet-Frebourg, N. et al. (2015) Detecting Genomic Signatures of Natural Selection with Principal Component Analysis: Application to the 1000 Genomes Data. Molecular Biology and Evolution 4, 1082-93.

[30] de Villemereuil, P. et al. (2014) Genome scan methods against more complex models: when and how much should we trust them? Molecular Ecology 23, 2006-2019.

[31] Vilas, A. et al. (2012) A simulation study on the performance of differentiation-based methods to detect selected loci using linked neutral markers. J Evol Biol 25, 1364-76.

[32] Novembre, J. and Barton, N. H. (2018) Tread Lightly Interpreting Polygenic Tests of Selection. Genetics 208, 1351-1355.

[33] Rosenberg, N. A. et al. (2019) Interpreting polygenic scores, polygenic adaptation, and human phenotypic differences. Evolution, Medicine, and Public Health 2019, 26-34.

[34] Berg, J. J. et al. (2019) Reduced signal for polygenic adaptation of height in UK Biobank. eLife 8, e39725.

[35] Sohail, M. et al. (2019) Polygenic adaptation on height is overestimated due to uncorrected stratification in genome-wide association studies. eLife 8, e39702.

[36] Uricchio, L. H. (2020) Evolutionary perspectives on polygenic selection, missing heritability, and GWAS. Human Genetics 139, 5-21.

[37] Csillery, K. et al. (2018) Detecting the genomic signal of polygenic adaptation and the role of epistasis in evolution. Molecular Ecology 27, 606-612. 
[38] Payne, J. L. and Wagner, A. (2019) The causes of evolvability and their evolution. Nature Reviews Genetics 20, 24-38.

[39] Manolio, T. A. et al. (2009) Finding the missing heritability of complex diseases. Nature 461, $747-753$.

[40] Boyle, E. A. et al. (2017) An Expanded View of Complex Traits: From Polygenic to Omnigenic. Cell 169, 1177-1186.

[41] Kudaravalli, S. et al. (2009) Gene expression levels are a target of recent natural selection in the human genome. Molecular Biology and Evolution 26, 649-658.

[42] Torgerson, D. G. et al. (2009) Evolutionary processes acting on candidate cis-regulatory regions in humans inferred from patterns of polymorphism and divergence. PLoS genetics 5, e1000592.

[43] Rougeux, C. et al. (2019) Polygenic selection drives the evolution of convergent transcriptomic landscapes across continents within a Nearctic sister species complex. Molecular Ecology 28, 4388-4403.

[44] Kirschner, M. W. (2005) The Meaning of Systems Biology. Cell 121, 503-504.

[45] Sonawane, A. R. et al. (2019) Network Medicine in the Age of Biomedical Big Data. Frontiers in Genetics 10.

[46] Wagner, A. (1994) Evolution of gene networks by gene duplications: a mathematical model and its implications on genome organization. Proceedings of the National Academy of Sciences 91, 4387-4391.

[47] Wagner, A. (1996) Does evolutionary plasticity evolve? Evolution 50, 1008-1023.

[48] Waddington, C. H. (1942) Canalization of development and the inheritance of acquired characters. Nature 150, 563-565.

[49] Siegal, M. L. and Bergman, A. (2002) Waddington's canalization revisited: Developmental stability and evolution. Proceedings of the National Academy of Sciences 99, 10528-10532.

[50] Runneburger, E. and Le Rouzic, A. (2016) Why and how genetic canalization evolves in gene regulatory networks. Bmc Evolutionary Biology 16. 
[51] Bergman, A. and Siegal, M. L. (2003) Evolutionary capacitance as a general feature of complex gene networks. Nature 424, 549-552.

[52] Rhone, B. et al. (2011) Impact of selection on genes involved in regulatory network: a modelling study. J Evol Biol 24, 2087-98.

[53] Bustamante, C. D. et al. (2005) Natural selection on protein-coding genes in the human genome. Nature 437, 1153-1157.

[54] Halfon, M. S. (2017) Perspectives on Gene Regulatory Network Evolution. Trends in Genetics 33, 436-447.

[55] Das Gupta, M. and Tsiantis, M. (2018) Gene networks and the evolution of plant morphology. Current Opinion in Plant Biology 45, 82-87.

[56] ten Tusscher, K. H. and Hogeweg, P. (2009) The role of genome and gene regulatory network canalization in the evolution of multi-trait polymorphisms and sympatric speciation. BMC Evolutionary Biology 9, 159.

[57] Doebley, J. F. et al. (2006) The Molecular Genetics of Crop Domestication. Cell 127, 13091321.

[58] Swanson-Wagner, R. et al. (2012) Reshaping of the maize transcriptome by domestication. Proceedings of the National Academy of Sciences 109, 11878-11883.

[59] Bullard, J. H. et al. (2010) Polygenic and directional regulatory evolution across pathways in Saccharomyces. Proceedings of the National Academy of Sciences 107, 5058-5063.

[60] MacNeil, L. T. and Walhout, A. J. M. (2011) Gene regulatory networks and the role of robustness and stochasticity in the control of gene expression. Genome Research 21, 645-657.

[61] Barghi, N. et al. (2019) Genetic redundancy fuels polygenic adaptation in Drosophila. PLOS Biology 17, e3000128.

[62] Albert, R. (2005) Scale-free networks in cell biology. Journal of Cell Science 118, 4947-4957.

[63] Platig, J. et al. (2016) Bipartite Community Structure of eQTLs. PLOS Computational Biology 12, e1005033. 
[64] Jordan, I. K. et al. (2004) Conservation and Coevolution in the Scale-Free Human Gene Coexpression Network. Molecular Biology and Evolution 21, 2058-2070.

[65] Josephs, E. B. et al. (2017) The Relationship between Selection, Network Connectivity, and Regulatory Variation within a Population of Capsella grandiflora. Genome Biology and Evolution 9, 1099-1109.

[66] Luisi, P. et al. (2015) Recent Positive Selection Has Acted On Genes Encoding Proteins With More Interactions Within the Whole Human Interactome. Genome Biology and Evolution 4, $1141-1154$.

[67] Fagny, M. et al. (2017) Exploring regulation in tissues with eQTL networks. PNAS 114, E7841-E7850.

[68] Baker, R. L. et al. (2019) Integrating transcriptomic network reconstruction and eQTL analyses reveals mechanistic connections between genomic architecture and Brassica rapa development. PLOS Genetics 15, e1008367.

[69] Barghi, N. et al. (2020) Polygenic adaptation: a unifying framework to understand positive selection. Nature Reviews Genetics 21, 769-781.

[70] Fisher, R. A. (1919) XV.-The Correlation between Relatives on the Supposition of Mendelian Inheritance. Transactions of the Royal Society of Edinburgh 52, 399-433.

[71] Mackay, T. F. C. et al. (2009) The genetics of quantitative traits: challenges and prospects. Nature Reviews Genetics 10, 565-577.

[72] Holland, J. B. (2007) Genetic architecture of complex traits in plants. Genome Studies and Molecular Genetics 10, 156-161.

[73] Timpson, N. J. et al. (2018) Genetic architecture: the shape of the genetic contribution to human traits and disease. Nature Reviews Genetics 19, 110-124.

[74] Barton, N. H. et al. (2017) The infinitesimal model: Definition, derivation, and implications. Theoretical Population Biology 118, 50 - 73.

[75] Peiffer, J. A. et al. (2014) The Genetic Architecture Of Maize Height. Genetics 196, 13371356. 
[76] Pais, T. M. et al. (2013) Comparative Polygenic Analysis of Maximal Ethanol Accumulation Capacity and Tolerance to High Ethanol Levels of Cell Proliferation in Yeast. PLOS Genetics 9, e1003548.

[77] Salome, P. A. et al. (2011) Genetic Architecture of Flowering-Time Variation in Arabidopsis thaliana. Genetics 188, 421.

[78] Fuchsberger, C. et al. (2016) The genetic architecture of type 2 diabetes. Nature advance online publication.

[79] Voight, B. F. et al. (2006) A map of recent positive selection in the human genome. PLoS Biol 4, e72.

[80] Ferrer-Admetlla, A. et al. (2014) On detecting incomplete soft or hard selective sweeps using haplotype structure. Mol Biol Evol 31, 1275-91. 
A

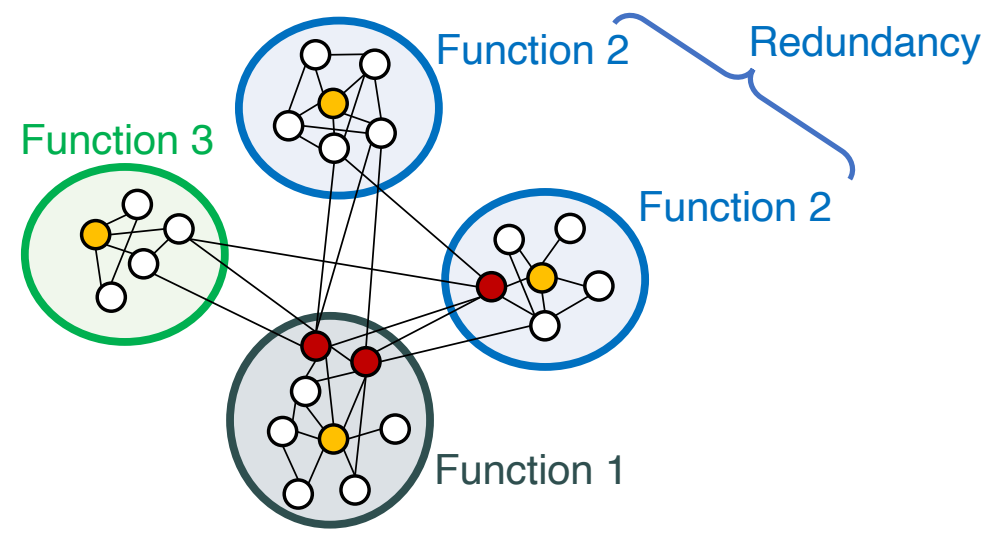

High-degree (pleiotropic, negative selection)

High core-score (negative/positive selection depending on environment stability

Peripheral (positive selection)
B

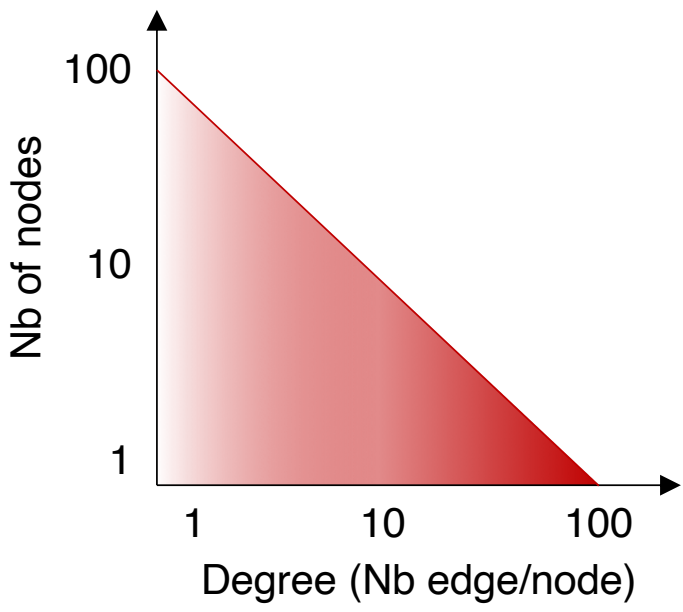

Figure 1: Impact of gene regulatory network structure on selection action. (A) Modular structure of a gene regulatory network. Genes are represented as circles and edges represent regulatory relationship between gene products. Genes are colored by topological characteristics: high degree genes in red, high core-score genes in yellow and others in white. The network is structured into modules, i.e. groups of genes that regulate each others. These modules often correspond to biological functions. Topological characteristics of gene regulatory networks such as redundancy (several modules involved in the regulation of the same biological functions) and pleiotropy (genes involved in the regulation of several biological functions) are highlighted. (B) Expected power law distribution of the node degree in $q$ gene regulatory network. 


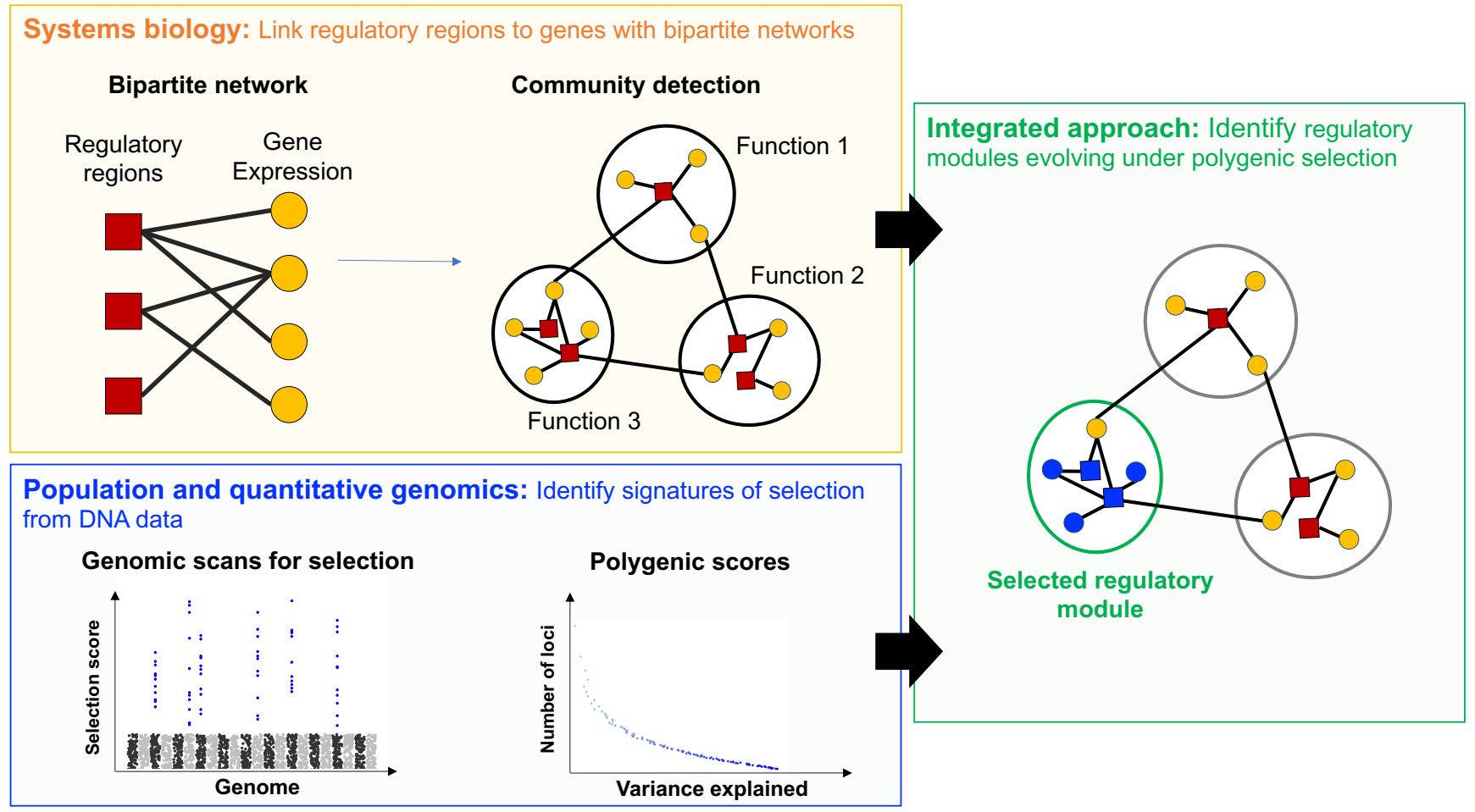

Figure 2: Proposition for a new integrated framework to detect signatures of polygenic selection. Genes and regulatory regions in blue present signatures of selection. 